\title{
Linfoma anaplásico de células grandes asociado a implantes mamarios. Importancia para el cirujano
}

\author{
Anaplastic large cell lymphoma associated with breast implants. Importance for the \\ surgeon
}

\author{
David Martínez-Ramos ${ }^{*}$, Laura Simón-Monterde², Consuelo Suelves-Piqueres ${ }^{1}$, Raquel Queralt-Martí y \\ Manuel Laguna-Sastre ${ }^{1}$ \\ ${ }^{1}$ Unidad de Patología Mamaria, Servicio de Cirugía General y del Aparato Digestivo, Hospital Universitario General de Castellón; ²Centro de Salud \\ Fernando El Católico, Castellón, España
}

\begin{abstract}
Resumen
El linfoma anaplásico de células grandes asociado a implantes mamarios (BIA-ALCL, breast implant associated-anaplastic large cell lymphoma) es una enfermedad infrecuente, pero el número de casos ha aumentado en los últimos años. Se puede presentar en la consulta del cirujano como un aumento del volumen mamario por derrame periprotésico o como masa capsular. El tratamiento de elección es la cirugía, con explante de las prótesis y exéresis de la cápsula periprotésica. El pronóstico, si el diagnóstico es precoz y el tratamiento es adecuado, es bueno. El objetivo del presente trabajo fue revisar la literatura científica existente hasta la fecha sobre el BIA-ALCL y poner de manifiesto la importancia que este tipo de neoplasia tiene para el cirujano.
\end{abstract}

PALABRAS CLAVE: Linfoma anaplásico. Células grandes. Implantes mamarios.

\begin{abstract}
Breast implant-associated anaplastic large-cell lymphoma (BIA-ALCL) is an infrequent disease, although the number of cases has increased in the recent years. BIA-ALCL is revealed during medical screening as an increase in the mammary volume due to periprosthetic effusion or as a capsular mass. Preferred treatment for BIA-ALCL is surgery, with explantation of the prostheses and exeresis of the periprosthetic capsule. Prognosis, after early diagnosis and adequate treatment, is good. The objective of the present work was to review the existing scientific literature on BIA-ALCL and to show the importance of this type of neoplasia for a successful treatment.
\end{abstract}

KEY WORDS: Lymphoma anaplastic. Cell large. Implants breast.

\section{Introducción}

La utilización de implantes mamarios es una práctica habitual en nuestro medio, donde estas prótesis pueden ser implantadas bien con fines estéticos (mamoplastias de aumento) o bien con fines reconstructivos, ya sea por cáncer de mama o por cirugías profilácticas de reducción de riesgo.

Como cualquier dispositivo implantable, las prótesis mamarias pueden presentar complicaciones, tales como contractura capsular, infecciones, hemorragias, seromas, fenómenos autoinmunitarios, etc. ${ }^{1}$ Hasta la

Correspondencia:

*David Martínez-Ramos

Hosp. Univ.General de Castellón

Avda. Benicassim, s/n

Fecha de recepción: 27-03-2018

Cir Cir. 2018;86:459-464

12004 Castellón, España

Fecha de aceptación: 14-05-2018

E-mail: doctormartinezramos@gmail.com

DOI: $10.24875 / C I R U .18000314$

Contents available at PubMed www.cirugiaycirujanos.com 
fecha, no se ha conseguido demostrar ninguna relación causal entre los implantes mamarios y el cáncer de mama. Sin embargo, desde hace aproximadamente dos décadas se está observando una preocupante asociación entre los implantes mamarios y un infrecuente tipo de linfoma: el linfoma anaplásico de células grandes. Este linfoma presenta unas características concretas y particulares, por lo que se ha denominado de manera específica linfoma anaplásico de células grandes asociado a implantes mamarios (BIA-ALCL, breast implant associated-anaplastic large cell lymphoma).

Cerca del $60 \%$ de los BIA-ALCL se producen en pacientes sometidas a aumentos mamarios primarios, pero un $40 \%$ ocurren en pacientes con reconstrucción mamaria ${ }^{2}$. Aunque es verdad que se trata de una situación infrecuente, el número de casos comunicados se ha incrementado exponencialmente en los últimos años. Además, como sucede en la mayor parte de las neoplasias, el pronóstico de este tipo de linfomas dependerá, en gran medida, de la posibilidad de realizar un tratamiento quirúrgico adecuado, lo que estará condicionado, asimismo, por un diagnóstico correcto y precoz. Por ello, el cirujano debe ser conocedor de esta situación, lo que le permitirá mantener un alto índice de sospecha.

El objetivo del presente trabajo fue hacer una puesta al día del estado de la situación sobre el BIA-ALCL y poner de manifiesto la importancia que este tipo de neoplasia tiene para el cirujano.

\section{Antecedentes históricos}

El linfoma anaplásico de células grandes (ALCL) fue descrito por primera vez en 1985 por Stein, et al. ${ }^{3}$ como un linfoma no Hodgkin de citología anaplásica y expresión del antígeno Ki-1 (CD30). Unos años más tarde, en 2008, la Organización Mundial de la Salud incluyó el ALCL en la clasificación de los linfomas, reconociendo dos variedades principales en función de la expresión de ALK (anaplastic lymphoma kinase): ALCL-ALK positivos y ALCL-ALK negativos ${ }^{4}$.

La primera asociación entre el ALCL y los implantes mamarios la establecieron en $1997 \mathrm{Keech}$, et al. ${ }^{5}$ en una serie de 46 casos diagnosticados en Australia y Nueva Zelanda. Desde entonces se han descrito diferentes casos y series de casos en la literatura, la mayor de ellas con menos de 200 casos incluidos ${ }^{6-12}$.

En el año 2011, la Food and Drug Administration (FDA) de los EE.UU. alertó sobre el posible riesgo de presentar un tipo de linfoma no Hodgkin asociado al uso de implantes mamarios, basándose en una serie de 60 casos comunicados en la literatura ${ }^{13}$. En su informe únicamente se advertía de una leve probabilidad de presentar ALCL en pacientes portadoras de prótesis mamarias, desaconsejando el explante de las prótesis si no existía sospecha de esta enfermedad. A partir de 2010 se instauraron en los EE.UU. unas medidas de alerta específica. En el último informe de la FDA, de febrero de $2017^{14}$, se habían registrado 359 casos en los EE.UU., aunque esta agencia reconocía que podía haber un sesgo de infranotificación, presentación de eventos duplicados y falta de información sobre el número total de implantes mamarios. Recientemente se ha propuesto incluir en la clasificación TNM el subtipo BIA-ALCL para las neoplasias linfoides ${ }^{15}$. En México y América Latina, el primer caso documentado fue el comunicado por Torres-Rivero, et al. ${ }^{16}$ en 2016 en una paciente de 43 años.

\section{Incidencia}

La incidencia exacta del BIA-ALCL es todavía desconocida por múltiples motivos. Por una parte, no existe un registro internacional de casos ni se trata de una enfermedad de declaración obligatoria, por lo que muchos casos pueden no haberse comunicado o, en el otro extremo, un único caso puede haberse publicado en diversas ocasiones por los diferentes especialistas que intervienen en el proceso diagnóstico-terapéutico. Por otra parte, aunque se estima que 10 millones de personas en el mundo son portadoras de implantes mamarios, no se dispone de datos exactos sobre esta cifra, por lo que todo se basa en estimaciones más o menos exactas. Por último, tenemos que admitir que existe todavía cierto desconocimiento sobre esta enfermedad entre los propios profesionales sanitarios, lo que podría conducir a su infradiagnóstico $y$, por tanto, a una incidencia falsamente baja ${ }^{17}$.

En este contexto necesariamente prudente, Doren, et al. ${ }^{18}$, basándose en 100 casos comunicados en los EE.UU., estimaron una incidencia de 2,03 casos por millón y año, aunque otros autores han aumentado sustancialmente esta estimación a un caso por cada 30,000 mujeres con prótesis por año $0^{19,20}$. De acuerdo con los datos disponibles, se estima que el riesgo de desarrollar un ALCL es 18,2-67,6 veces mayor en las mujeres con prótesis mamarias que en las mujeres sin implantes. Sin embargo, en un estudio de cohortes de base poblacional danés con cerca de 20,000 mujeres portadoras de prótesis mamarias no se 
encontró ningún caso de BIA-ALCL ${ }^{21}$. Como se ha dicho, estos datos son muy indirectos y no pueden considerarse definitivos.

\section{Patogenia}

La etiopatogenia de esta enfermedad y sus factores de riesgo no se conocen con exactitud, aunque los últimos datos apuntan a que su aparición estaría relacionada con un proceso inflamatorio local ${ }^{17,22}$. De este modo, podría hipotetizarse que las partículas de silicona o la superficie del implante iniciarían el proceso inflamatorio que conduciría al desarrollo del linfoma. Para otros autores, podría ser secundario a la estimulación linfocítica por una sobreinfección en el biofilm de la prótesis. De hecho, $\mathrm{Hu}$, et al. ${ }^{23}$ comunicaron un aumento de la prevalencia de Ralstonia spp. (un microorganismo oportunista gramnegativo) en las cápsulas de las pacientes con BIA-ALCL cuando se compararon con controles. Podría suceder algo parecido a lo que ocurre con los linfomas tipo MALT, en los que la inflamación crónica por Helicobacter pylori está bien documentada. Otras teorías apuntan a fenómenos autoinmunitarios, predisposición genética 0 antecedentes de traumatismos capsulares ${ }^{19}$. Así los datos, todavía es pronto para asumir como válida cualquiera de estas hipótesis (o la combinación de ellas) y se necesitan más estudios que permitan establecer mecanismos etiopatogénicos más precisos.

\section{Tipos de implantes protésicos implicados}

Los primeros casos de ALCL se relacionaron tanto con los implantes lisos como con los texturizados, aunque recientemente se ha comunicado que las prótesis texturizadas se relacionan con mayor frecuencia con el BIA-ALCL ${ }^{18}$. Asimismo, tanto los implantes de silicona como los de solución salina se han relacionado con la aparición del linfoma, con un predominio en las prótesis de silicona (39 frente a $61 \%)^{17,19}$. No obstante, conviene considerar que, especialmente en los casos de cirugía reconstructiva, muchas pacientes han sido portadoras de diferentes implantes y recambios (expansores o prótesis definitivas), por lo que resulta difícil establecer el tipo de prótesis causante. Además, el proceso de texturización de las prótesis varía de unas casas comerciales a otras, por lo que todavía es más complicado establecer asociaciones concluyentes. Por otro lado, se han comunicado casos de diferentes casas comerciales, aunque casi todos los estudios se han realizado en los EE.UU., por lo que las marcas comercializadas de ese país son las que se han estudiado con más detenimiento.

\section{Sospecha diagnóstica y manifestaciones clínicas}

Existen dos formas fundamentales de presentación del BIA-ALCL y que deben alertar al cirujano. Por un lado, la forma de presentación clínica más frecuente es como un seroma periprotésico tardío recidivante (con aumento brusco del volumen mamario), y la segunda es como una masa en la cápsula periprotési$\mathrm{ca}^{24,25}$. Otras manifestaciones menos frecuentes han sido la adenopatía axilar palpable, la contractura capsular, las lesiones cutáneas ${ }^{26}$ y los síntomas tipo B (fiebre, adenopatías, sudoraciones nocturnas y fatiga). Casi el $15 \%$ de los casos presentan metástasis en los ganglios axilares, con muy poca frecuencia bilaterales, con menos de 10 casos comunicados ${ }^{27}$.

En la revisión sistemática llevada a cabo por Leberfinger, et al. ${ }^{20}$, la media de edad para la aparición del ALCL fue de 51 años, siendo las mujeres con reconstrucción de mayor edad que las operadas con fines estéticos (57 frente a 46 años de media, respectivamente), posiblemente porque las mujeres operadas con fines estéticos son más jóvenes de inicio. Es importante destacar que, en la mayoría de los países de nuestro entorno, estos rangos de edad se incluyen en los programas de cribado poblacional, lo que debería tenerse en cuenta también en estas mujeres durante el estudio de cribado. Por otra parte, la media de tiempo desde el implante de la prótesis hasta la aparición del linfoma es de alrededor de 10 años en la mayoría de las series ${ }^{2,20}$.

\section{Diagnóstico}

La primera exploración complementaria ante una paciente portadora de prótesis mamaria que presenta un aumento brusco del volumen mamario suele ser una ecografía, que permitirá identificar la presencia de líquido periprotésico, así como la existencia de masas capsulares. Además, con la ecografía se puede dirigir la punción-aspiración de líquido periprotésico para su análisis. El aspecto de este líquido suele ser turbio en caso de BIA-ALCL. El análisis citológico del líquido extraído orientará al diagnóstico, que se confirmará mediante análisis inmunohistoquímico. Obviamente, es importante que el cirujano sospeche esta enfermedad para que solicite un estudio inmunohistoquímico dirigido del líquido, ya que los 
BIA-ALCL son positivos para CD30, positivos para antígenos de membrana epitelial y ALK negativos. La biopsia capsular no está indicada ante cualquier derrame periprotésico, pero la cápsula sí debe analizarse histopatológicamente cuando se realiza una cirugía de exéresis de esta. Del mismo modo, si se aprecia un ganglio linfático sospechoso durante la cirugía, debería extirparse para su estudio ${ }^{27}$.

Se han propuesto diversos algoritmos de manejo ante la sospecha de un BIA-ALCL, según diferentes países y sistemas sanitarios ${ }^{2,27,28}$, aunque todos coinciden en la necesidad de realizar una ecografía ante la sospecha y de hacer un estudio citológico del líquido periprotésico. Otras pruebas de imagen, en especial la resonancia magnética, también pueden ser útiles. Así, según los resultados de Adrada, et al. ${ }^{29}$, la sensibilidad para detectar el derrame fue del $84 \%$ para la ecografía, del $82 \%$ para la resonancia magnética, del $55 \%$ para la tomografía computarizada (TC) y del $38 \%$ para la tomografía por emisión de positrones (PET). La sensibilidad de la mamografía para la detección de alguna anormalidad (sin distinguir entre seroma y masa) fue del $73 \%$, mientras que la especificidad fue del $50 \%$. Conviene apuntar que estos datos se obtuvieron al analizar los resultados de 40 casos, algunos de ellos de la literatura (no de los propios autores), con las limitaciones que ello implica.

Por último, el estudio de extensión de enfermedad sistémica de elección debería hacerse mediante PET/ $\mathrm{TC}$, idealmente después de la cirugía (una vez extirpada la lesión) para disminuir el riesgo de falsos positivos $^{27,30}$.

\section{Estadificación}

El linfoma anaplásico de células grandes sistémico (no asociado a implantes mamarios) es un linfoma infrecuente y agresivo, con un pronóstico infausto, que requiere tratamiento sistémico intensivo y que se estadifica generalmente mediante la clasificación de Ann Arbor ${ }^{31}$. Esta clasificación, sin embargo, podría no ser adecuada para el BIA-ALCL, puesto que sobreestadificaría y, por tanto, se sobretrataría a estas pacientes que, en principio, tienen mucho mejor pronóstico ${ }^{2}$. Por este motivo, Clemens, et al..$^{15}$ han propuesto una modificación específica para el BIA-ALCL que reconoce un grupo de muy bajo riesgo con enfermedad intraluminal y lo diferencia de un segundo grupo de alto riesgo con enfermedad extraluminal que se beneficiarían de un tratamiento sistémico.

\section{Tratamiento}

Lo más importante para el cirujano es saber que, a diferencia de la mayoría de los linfomas, el BIA-ALCL a menudo es curable exclusivamente con cirugía ${ }^{19}$. Aunque no existe un protocolo de tratamiento establecido, lo que parece estar claro es que la cirugía es el tratamiento de elección cuando la enfermedad está localizada ${ }^{15,27}$, lo que ocurre en la mayoría de los casos. Según la evidencia científica actual, el tratamiento óptimo debe incluir el explante de la prótesis, la capsulectomía completa, la exéresis de todos los implantes de linfoma con márgenes (en los casos infiltrativos T2 y T3) y la exéresis de cualquier adenopatía sospechosa ${ }^{19}$. En la actualidad no se tienen datos para recomendar la mastectomía, la biopsia selectiva del ganglio centinela ni la linfadenectomía axilar. Es importante valorar la mama contralateral, puesto que cerca del $5 \%$ de los casos presentaban linfoma en la cápsula, por lo que debería tomarse la decisión de acuerdo con la paciente convenientemente informada. No se recomienda la colocación de una nueva prótesis tras el diagnóstico de un BIA-ALCL y no se dispone de datos para hacer recomendaciones sobre una eventual reconstrucción mamaria posterior ${ }^{15,25}$.

En cuanto a los tratamientos adyuvantes, existe poca evidencia para los casos en que no es posible la exéresis completa de las lesiones o que tienen enfermedad a distancia, ya que no hay ensayos clínicos disponibles. Si hay afectación de los márgenes quirúrgicos, enfermedad irresecable o invasión torácica, puede ser beneficiosa la radioterapia. Si existe enfermedad diseminada, el tratamiento sistémico está indicado mediante esquemas similares a los del linfoma $T$ periférico, aunque los datos son poco concluyentes y se necesitan nuevos estudios. El protocolo de quimioterapia más extendido para el tratamiento es el esquema CHOP (ciclofosfamida, clorhidrato de doxorubicina, sulfato de vincristina y prednisona). No obstante, ante la falta de suficiente evidencia científica, parece clara la necesidad de individualizar los tratamientos en espera de nuevos estudios ${ }^{19}$. Recientemente, Leberfinger, et al. ${ }^{20}$ han propuesto un algoritmo de manejo de estas pacientes, cuya adaptación y traducción se muestra en la figura 1.

\section{Pronóstico}

El pronóstico de este tipo de linfoma es muy favorable, excepto en algunos casos que pueden llegar a ser 


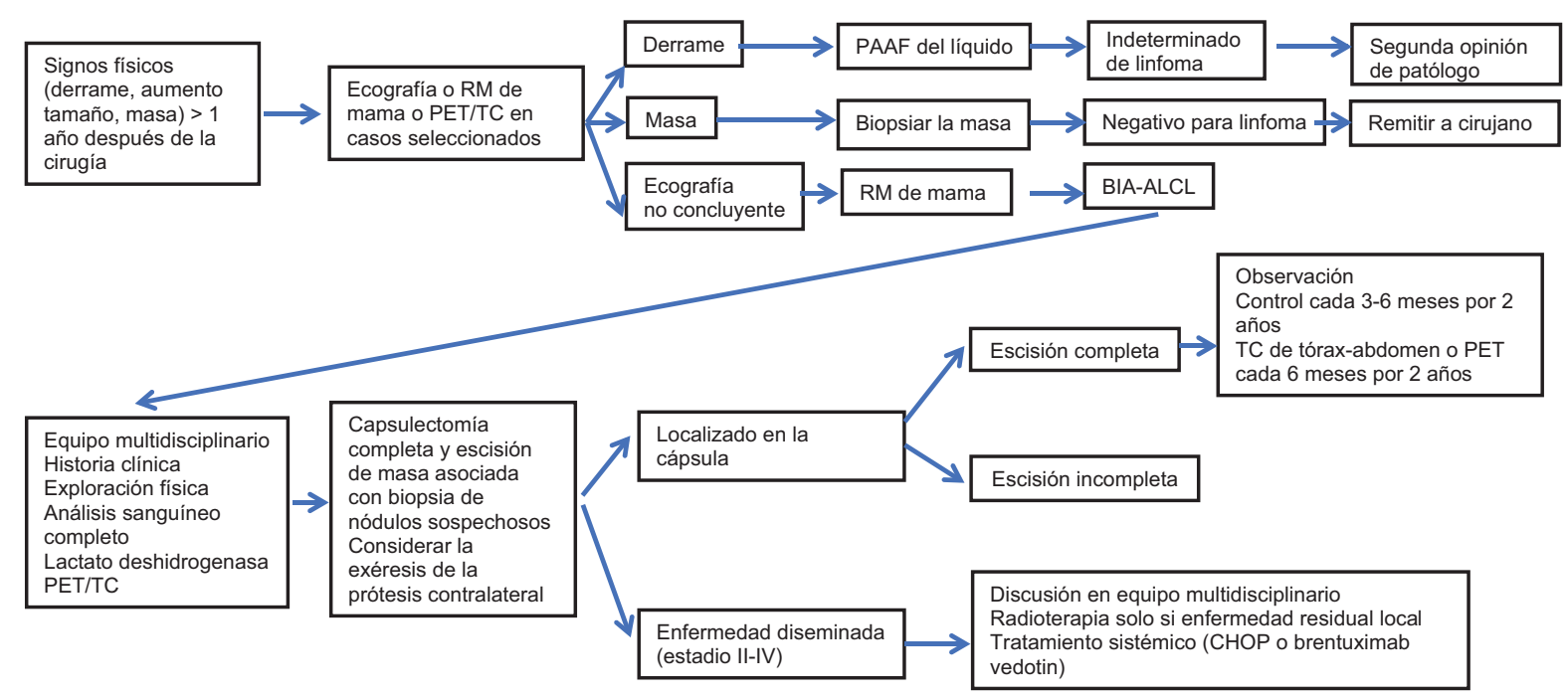

Figura 1. Algoritmo propuesto y adaptado de Leberfinger et al. ${ }^{20}$ para el diagnóstico y el tratamiento del linfoma anaplásico de células grandes asociado a implantes mamarios. BIA-ALCL: breast implant-associated anaplastic large cell lymphoma; CHOP: ciclofosfamida, hidroxidaunorubicina, vincristina, prednisona; PAAF: punción-aspiración con aguja fina; PET: tomografía por emisión de positrones; RM: resonancia magnética; TC: tomografía computarizada.

especialmente agresivos. Las lesiones nodulares, la afectación de los ganglios axilares, la afectación bilateral y el patrón infiltrativo en la histología de la cápsula se han relacionado con un comportamiento más agresivo. El pronóstico es mejor con cirugía de exéresis completa de la cápsula que con resecciones parciales, tanto en supervivencia global como en intervalo libre de enfermedad ${ }^{15}$. Algunos autores han considerado que la presencia de linfoma en el líquido periprotésico es una situación diferente al tumor con masa infiltrante, pero no existe suficiente evidencia para tal aseveración $^{17}$. En la mayoría de las series el tiempo de seguimiento todavía es corto, aunque algunos autores han comunicado seguimientos de hasta 20 años $^{32}$. La tasa de recurrencia actual con la exéresis completa de las lesiones es del 6-11\%, y la supervivencia media global ha sido de 12-13 años ${ }^{19}$. Hasta el momento, de los 359 casos comunicados a la FDA, nueve fallecieron como consecuencia de su enfermedad ${ }^{14}$.

En cuanto al seguimiento, según las recomendaciones de la National Comprehensive Cancer Network ${ }^{27}$, si la exéresis del tumor ha sido completa y no hay enfermedad residual debería hacerse cada 3-6 meses mediante anamnesis y exploración física durante al menos 2 años. En estos 2 años debería añadirse una TC torácica, abdominal y pélvica con contraste, o una PET-TC, cada 6 meses. Transcurridos los 2 años, el seguimiento dependerá de las manifestaciones clínicas de la paciente ${ }^{27}$.

\section{Consentimiento informado}

Nadie cuestiona ya que el consentimiento informado es una parte más de cualquier acto quirúrgico. Es un derecho de cualquier paciente el disponer de toda la información necesaria para una toma de decisiones adecuada, así como de los posibles riesgos de la cirugía a la que va a someterse. Por este motivo, la literatura consultada coincide de manera unánime en que el BIA-ALCL es una enfermedad infrecuente, pero de la que se debería informar a todas las pacientes candidatas a colocación de prótesis ${ }^{2,33}$. Los objetivos de esta información son tres: 1) informar a las pacientes de la existencia de esta rara enfermedad; 2) informar de la forma de presentación habitual para poder realizar un diagnóstico precoz; y 3) informar a las pacientes de las opciones de tratamiento existentes en caso de que se presente.

\section{Conclusión}

El BIA-ALCL es una enfermedad infrecuente, pero el número de casos se ha incrementado en los últimos años. Es importante que el cirujano y el especialista en mama lo conozcan, porque un diagnóstico y un tratamiento precoces pueden suponer la curación de la paciente. 


\section{Bibliografía}

1. Rocco N, Rispoli C, Moja L, Amato B, lannone L, Testa S, et al. Different types of implants for reconstructive breast surgery. Cochrane Database Syst Rev. 2016;(5):CD010895.

2. Johnson L, O'Donoghue JM, McLean N, Turton P, Khan AA, Turner SD Breast implant associated anaplastic large cell lymphoma: the UK experience. Recommendations on its management and implications for informed consent. Eur J Surg Oncol. 2017;43:1393-401.

3. Stein H, Mason DY, Gerdes J, O'Connor N, Wainscoat J, Pallesen G et al. The expression of the Hodgkin's disease associated antigen Ki-1 in reactive and neoplastic lymphoid tissue: evidence that Reed-Sternberg cells and histiocytic malignancies are derived from activated lymphoid cells. Blood. 1985;66:848-58.

4. Sathyanarayanan V, Lokesh KN, Lakshmaiah KC, Govind Babu K Lokanatha D, Suresh Babu MC, et al. Anaplastic lymphoma kinase (ALK) positive anaplastic large cell lymphoma (ALCL) of breast in a patient without a breast implant. Ann Saudi Med. 2014;34:551-4.

5. Keech JA Jr, Creech BJ. Anaplastic T-cell lymphoma in proximity to a saline-filled breast implant. Plast Reconstr Surg. 1997;100:554-5.

6. Nogueira Batista B, Garicochea B, Nunes Aguilar VL, Carvalho FM, Millan LS, Pires Fraga MF. Report of a case of anaplastic large cell lymphoma associated with a breast implant in a Brazilian patient. Rev Bras Cir Plast 2017:32:445-9.

7. Letter H, Rop B, Edison MN, Turner P. Breast implant-associated anaplastic large cell lymphoma: a case report and literature review. Cureus 2016;8:e546.

8. Hwang MJ, Brown H, Murrin R, Momtahan N, Sterne GD. Breast implant-associated anaplastic large cell lymphoma: a case report and literature review. Aesthetic Plast Surg. 2015;39:391-5.

9. Evren S, Khoury T, Neppalli V, Cappuccino H, Hernández-llizaliturri FJ, Kumar P. Breast implant-associated anaplastic large cell lymphoma (ALCL): a case report. Am J Case Rep. 2017;18:605-10.

10. Zimmerman A, Locke FL, Emole J, Rosa M, Horna P, Hoover S, et al. Recurrent systemic anaplastic lymphoma kinase-negative anaplastic large cell lymphoma presenting as a breast implant-associated lesion. Cancer Control. 2015;22:369-73.

11. Popplewell L, Thomas $\mathrm{SH}$, Huang $\mathrm{Q}$, Chang $\mathrm{KL}$, Forman SJ. Primary anaplastic large-cell lymphoma associated with breast implants. Leuk Lymphoma. 2011;52:1481-7.

12. Xu J, Wei S. Breast implant-associated anaplastic large cell lymphoma: review of a distinct clinicopathologic entity. Arch Pathol Lab Med. 2014;138:842-6

13. Center for Devices and Radiological Health U.S. Food and Drug. Anaplastic large cell lymphoma (ALCL) in women with breast implants: preliminary FDA findings and analyses. 2011. (Consultado el 14 de diciembre de 2017.) Disponible en: https://www.fda.gov/MedicalDevices/ ProductsandMedicalProcedures/ImplantsandProsthetics/Breastlmplants/ ucm239995.htm.

14. Center for Devices and Radiological Health U.S. Food and Drug. Breast implant-associated anaplastic large cell lymphoma (BIA-ALCL). 2017. (Consultado el 14 de diciembre de 2017.) Disponible en: https://www. fda.gov/medicaldevices/productsandmedicalprocedures/implantsandprosthetics/breastimplants/ucm239995.htm.

15. Clemens MW, Medeiros LJ, Butler CE, Hunt KK, Fanale MA, Horwitz S, et al. Complete surgical excision is essential for the management of patients with breast implant-associated anaplastic large-cell lymphoma. J Clin Oncol. 2016;34:160-8.

16. Torres-Rivero C, Ramos-Gallardo G, Nambo-Lucio MJ, Vaquero-Pérez MM. Primer caso en México y América Latina de linfoma anaplásico de células gigantes en paciente con implantes mamarios. Cir Plast. Iberolatinoam. 2016:42:175-80.

17. O'Neill AC, Zhong T, Hofer SOP. Implications of breast implant-associated anaplastic large cell lymphoma (BIA-ALCL) for breast cancer reconstruction: an update for surgical oncologists. Ann Surg Oncol. 2017;24: 3174-9.

18. Doren EL, Miranda RN, Selber JC, Garvey PB, Liu J, Medeiros LJ, et al U.S. epidemiology of breast implant-associated anaplastic large cell lymphoma. Plast Reconstr Surg. 2017;139:1042-50.

19. Kaartinen I, Sunela K, Alanko J, Hukkinen K, Karjalainen-Lindsberg ML, Svarvar C. Breast implant-associated anaplastic large cell lymphoma — from diagnosis to treatment. Eur J Surg Oncol. 2017;43:1385-92.

20. Leberfinger AN, Behar BJ, Williams NC, Rakszawski KL, Potochny JD, Mackay DR, et al. Breast implant-associated anaplastic large cell lymphoma: a systematic review. JAMA Surg. 2017;152:1161-8.

21. Vase MØ, Friis S, Bautz A, Bendix K, Sørensen HT, d'Amore F. Breast implants and anaplastic large-cell lymphoma: a Danish population-based cohort study. Cancer Epidemiol Biomarkers Prev. 2013;22:2126-9.

22. George EV, Pharm J, Houston C, Al-Quran S, Brian G, Dong H, et al. Breast implant-associated ALK-negative anaplastic large cell lymphoma: a case report and discussion of possible pathogenesis. Int J Clin Exp Pathol. 2013:6:1631-42.

23. $\mathrm{Hu} \mathrm{H}$, Johani K, Almatroudi A, Vickery K, Van Natta B, Kadin ME, et al. Bacterial biofilm infection detected in breast implant-associated anaplastic large-cell lymphoma. Plast Reconstr Surg. 2016;137:1659-69.

24. Fernández-Sobrino I, Cordones-Guerrero JJ, Benítez-Dupin O, Cornejo-Ladrero Jl, Lobo-Samper F. Palpable mass as an atypical presentation of breast implants-associated anaplastic large cell lymphoma. Cir Plast berolatinoam. 2017;43:129-35.

25. Clemens MW, Nava MB, Rocco N, Miranda RN. Understanding rare adverse sequelae of breast implants: anaplastic large-cell lymphoma, late seromas, and double capsules. Gland Surg. 2017:6:169-84.

26. Alcalá R, Llombart B, Lavernia J, Traves V, Guillén C, Sanmartín O. Skin involvement as the first manifestation of breast implant-associated anaplastic large cell lymphoma. J Cutan Pathol. 2016;43:602-8.

27. Clemens MW, Horwitz SM. NCCN consensus guidelines for the diagnosis and management of breast implant-associated anaplastic large cell lymphoma. Aesthet Surg J. 2017;37:285-9.

28. Ramos-Gallardo G, Cuenca-Pardo J, Rodríguez-Olivares E, Iribarren-Moreno R, Contreras-Bulnes L, Vallarta-Rodríguez A, et al. Breast implant and anaplastic large cell lymphoma meta-analysis. J Invest Surg. 2017;30:56-65.

29. Adrada BE, Miranda RN, Rauch GM, Arribas E, Kanagal-Shamanna R, Clemens MW, et al. Breast implant-associated anaplastic large cell lymphoma: sensitivity, specificity, and findings of imaging studies in 44 patients. Breast Cancer Res Treat. 2014:147:1-14.

30. Acevedo-Báñez I, García-Gómez FJ, Jiménez-Granero $P$, Carrillo-Cruz E, Pérez-López O, Borrego-Dorado I. 18F-FDG-PET/CT in implant-associated anaplastic large cell lymphoma of the breast. $\mathrm{Br} \mathrm{J}$ Haematol. 2015;169:1.

31. Laurent C, Delas A, Gaulard P, Haioun C, Moreau A, Xerri L, et al. Breast implant-associated anaplastic large cell lymphoma: two distinct clinicopathological variants with different outcomes. Ann Oncol. 2016;27:306-14.

32. Miranda RN, Aladily TN, Prince HM, Kanagal-Shamanna R, de Jong D, Fayad LE, et al. Breast implant-associated anaplastic large-cell lymphoma: long-term follow-up of 60 patients. J Clin Oncol. 2014;32:114-20.

33. Clemens MW, Miranda RN, Butler CE. Breast implant informed consent should include the risk of anaplastic large cell lymphoma. Plast Reconstr Surg. 2016;137:1117-22. 